\title{
A temperature-independent force transducer using one optical fiber with multiple Bragg gratings
}

\author{
Ruiya Lia), Yuegang Tan, Liu Hong, Zude Zhou, Tianliang Li, \\ and Li Cai \\ School of Mechanical and Electrical Engineering, Wuhan University of Technology, \\ 122 Luoshi Road, Wuhan, Hubei, P.R.China \\ a) liruiya@whut.edu.cn
}

\begin{abstract}
Typical electrical or piezoelectric force sensors may fail in the industrial applications with strong electromagnetic interference (EMI). To address this issue, this paper develops a new temperature-independent force transducer based on theories of elastic cantilever beam and Bragg wavelength shift in fiber Bragg gratings. The detailed design of the structure and theoretical analysis are given to introduce the measurement principle of the transducer and temperature compensation of fiber Bragg gratings (FBG). Extensive experiments have been conducted to evaluate the performance of the developed force transducer. Experimental results demonstrate that the developed FBG force transducer has a force sensitivity $84.43 \mathrm{pm} / \mathrm{kN}$ within a measured range from 0 to $50 \mathrm{kN}$, which is consistent with the theoretical sensitivity $87.77 \mathrm{pm} / \mathrm{kN}$. Moreover, experimentally the transducer also achieves good linearity, repeatability and temperature independency. The developed force transducer can assist in monitoring the states of heavy-duty machines in the harsh industrial environment.
\end{abstract}

Keywords: FBG (fiber Bragg grating), force transducer, temperature compensation

Classification: Optical systems

\section{References}

[1] D. M. Ştefănescu and M. A. Anghel: Measurement 46 (2013) 949 (DOI: 10.1016/j.measurement.2012.10.020).

[2] Y. Wei and Q. Xu: Sens. Actuators A Phys. 234 (2015) 359 (DOI: 10.1016/ j.sna.2015.09.028).

[3] Z. Yang, et al.: Sens. Actuators A Phys. 136 (2007) 304 (DOI: 10.1016/j.sna. 2006.10.026).

[4] L. Cai, et al.: IEICE Electron. Express 12 (2015) 20150271 (DOI: 10.1587/ elex.12.20150271).

[5] S. J. Mihailov: Sensors 12 (2012) 1898 (DOI: 10.3390/s120201898).

[6] X. Li, et al:: Sens. Actuators A Phys. 223 (2015) 105 (DOI: 10.1016/j.sna. 2015.01.003).

[7] D. Jiang, et al.: Instrum. Tech. Sensor 2 (2006) 43. 
[8] T. Guo, et al.: J. Lightwave Technol. 24 (2006) 3797 (DOI: 10.1109/JLT.2006. 881841).

[9] J. Huang, et al.: Adv. Mech. Eng. 5 (2013) 125404 (DOI: 10.1155/2013/ 125404).

[10] W. Zhou, et al.: Microw. Opt. Technol. Lett. 52 (2010) 2020 (DOI: 10.1002/ mop.25397).

[11] B. Dong, et al.: Sens. Actuators A Phys. 147 (2008) 169 (DOI: 10.1016/j.sna. 2008.05.019).

[12] S. P. Timoshenko and J. N. Goodier: Theory of Elasticity (McGraw-Hill, New York, 1970) 3rd ed. 42.

[13] Y. J. Rao: Opt. Fiber Sens. Technol. 2 (1998) 355 (DOI: 10.1007/978-1-46155787-6_11).

\section{Introduction}

Nowadays, intelligent engineering systems require monitoring the states of machines to assist in the optimization of production quality and efficiency. Therefore, the development of precise, cost-effective and reliable sensing system is crucial in industrials. Traditional force transducers using resistive foils or piezoelectric materials are mainly based on Wheatstone bridge circuit and piezoelectric effect [1, $2,3]$. However, the measured signals from such electrical transducers are susceptible to be contaminated by the electromagnetic interference (EMI), which is common in the harsh industrial environment. Because the advantages such as anti-electromagnetic interference, anti-oil corrosion and multiple measuring points in one optical fiber, fiber Bragg grating (FBG) based transducer have attracted a lot of interests and been widely investigated by researchers and industrial engineers recently $[4,5]$.

The most popular spring element for FBG based force transducer is ring-type, which used a ring-type structure as the spring element and FBGs were installed on the outside surface of the ring to sense the strain. Then, the relationship between the strain of the structure and the measured force was established through mechanics of elasticity. Literatures $[6,7]$ both presented ring-type force sensors for bridge cable force monitoring, with FBG strain sensors stocked to the outside surface of the ring. The measured range of ring-type force sensor is large, but it does not have a high sensitivity. In order to improve the sensitivity of FBG force transducers, researchers have optimized the spring element structure of the transducer and mounted the FBGs on the spring element structure where generates the largest strain, mostly the stress concentration points. For instance, Tuan Guo et al. presented a beam-type force sensor with FBG glued at the fixed end of the beam [8]; Jun Huang et al. proposed a fiber Bragg grating tension sensor for anchor rope. The spring element of the sensor is a pull rod with two holes, and the FBGs are stocked at the edge of the holes [9].

Although the length of Bragg gratings can be made lesser than $3 \mathrm{~mm}$, however, too short Bragg grating region may reduce the measuring accuracy of FBG. Therefore in industrials, the most popular length of FBG is $10 \mathrm{~mm}$. With such a long Bragg grating region pasted at stress concentration place, disregarding of the 
high strain gradient at the Bragg grating region of the FBG is obviously unreasonable. At present, few scholars discussed this problem.

Moreover, the temperature cross-sensitivity of FBG can cause inaccurate measurements. A number of techniques such as using a reference FBG, writing two FBGs in different diameter fibers, using a strain chirped FBG, have been proposed $[7,10,11]$. While the reference $\mathrm{FBG}$ method have poor temperature compensation accuracy as the installation patterns are always different between the FBG to sense the strain and FBG to sense the temperature; method of writing two FBGs in different diameter fibers is strict to the micro machining process; the strain chirped FBG has only small measured range and are easy to be destroyed.

To address those issues above, a new spoke-type FBG force transducer has been developed and studied in this work. The theoretical model for the FBG force transducer is derived, considering non-uniform load on different spokes and nonuniform strain distribution in the FBG grating region. First, accurate two-dimensional plane strain distribution of the surface to be glued FBG on is analyzed. Then, the theoretical strain detected by FBG is calculated by curvilinear integral algorithm. The theoretical calculation results and experiment results shows high concordance. Using the FBGs' wavelength shift difference as sensing signal, the force sensitivity is improved and the temperature cross-sensitivity is effectively avoided. As 6 FBGs are connected in one fiber, this kind of FBG force transducer could be widely used for quasi-distributed force measurement.

\section{Principle and sensitization process model of the sensor}

The proposed FBG force transducer is based on a spoke structure. Its detailed schematics are illustrated in Fig. 1. The force transducer mainly consists of 6 FBGs (imprinted in one fiber) and a 3-spoke-type spring element. Every two FBGs (FBG $i 1$, FBG $i 2, i=1,2,3$ ) are bonded on the two side-surfaces of the spoke $i$ with epoxy adhesive as shown in Fig. 1(a). FBG $i 1$ and FBG $i 2$ are at the middle height of spoke $i$ with an identical angle $+135^{\circ}$ and $+45^{\circ}$ respectively along the direction of the neutral axis of the spoke $i$ as shown in Fig. 1(b). The spokes convert linearly the force into strain, and the 6 FBGs are used to detect the strain distributed on the side-surface of the spokes. It is worth noting that FBGs are not stocked on the top and bottom surface of the spoke because the stress gradient at top and bottom surface is very large, which easily cause FBG chirping. In addition, just three spokes are used but not four or even more, because three spokes are enough to support a plane, the structure of four spokes will increase the number of FBGs, which is not necessary.

As shown in Fig. 1(b) and Fig. 1(c), deformation and strain would be produced on the spokes when force $F$ to be measured is applied on the inner shell. Force sustained by spokes $i$ can be represented by $F_{i}(i=1,2,3)$. In practical application, forces sustained by different spokes are not necessarily uniform, thus it is not essential that $F=3 F_{1}=3 F_{2}=3 F_{3}$. While force $F$ is equal to the sum of $F_{i}$ $(i=1,2,3)$ : 


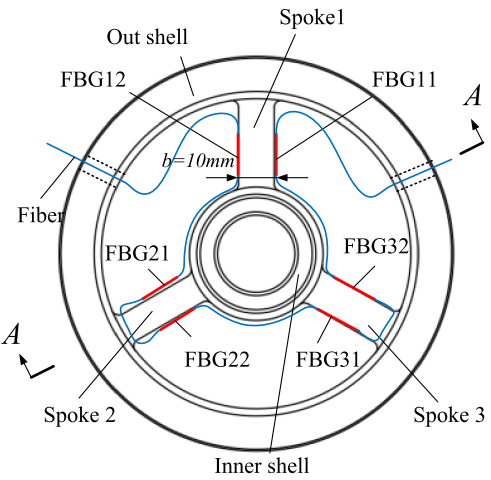

(a) Top view

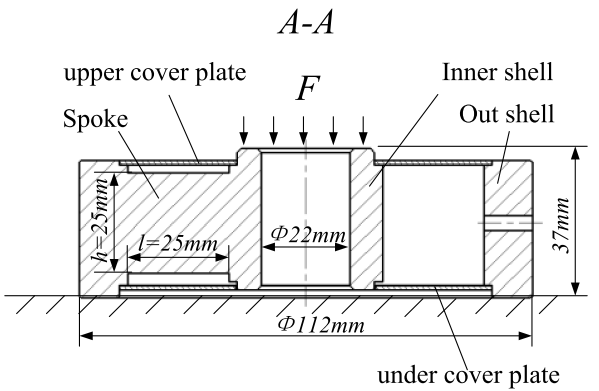

(c) Front sectional view

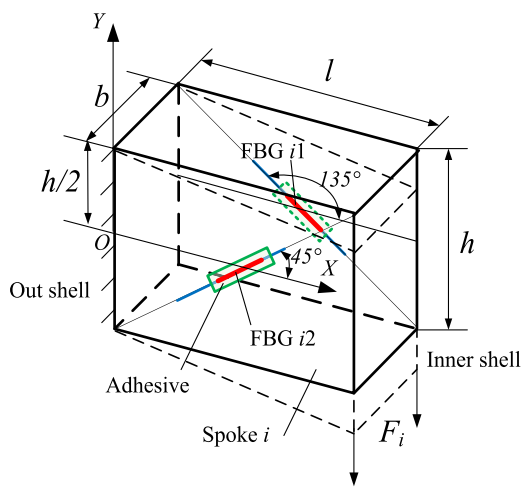

(b) Position of FBG on the spoke

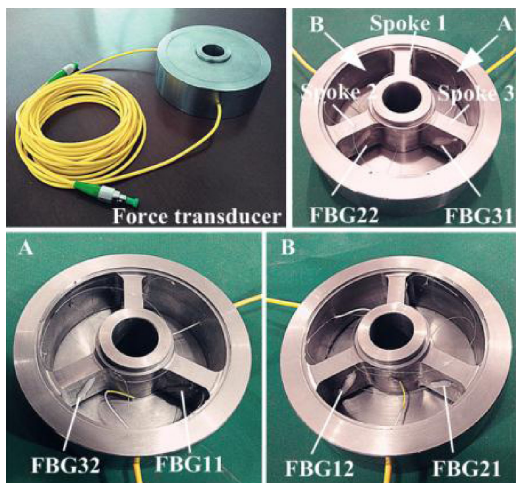

(d) Images of real force transducer

Fig. 1. The proposed structure with FBGs frame.

$$
F=\sum_{i=1}^{3} F_{i}
$$

Assuming the outer shell fixed, each spoke can be considered as an elastic cantilever beam. As shown in Fig. 1(b), according to the elastic theory, the stress in $\mathrm{Y}$ direction is zero, thus $\sigma_{y}=0$, the stress in $\mathrm{X}$ direction $\sigma_{x}$ and the shear stress $\tau_{x y}$ in XOY plane can be calculated by the following [12]:

$$
\begin{gathered}
\sigma_{x}=\frac{F_{i}}{I}(l-x) y \\
\tau_{x y}=\frac{F_{i}}{I}\left(\frac{h^{2}}{8}-\frac{y^{2}}{2}\right)
\end{gathered}
$$

Where $F_{i}$ represents the force loaded on spokes $i, I=b h^{3} / 12$ is the inertia moment of the cross section, $l$ is the total length of the spoke, $b$ is the width of the spoke, $h$ is the total height of the spoke. Based on Eq. (3), it can be known that the shear stress $\tau_{x y}$ shows parabola distribution in y-axis direction, and in the location of neutral layer the shear stress is the largest. By Eqs. (2), (3) and $\sigma_{y}=0$, the neutral layer is in a state of pure shear stress. According to the principle of elastic mechanics [12], for the same spoke, at neutral layer, the normal stress in the direction of $+135^{\circ}$ and $+45^{\circ}$ along the neutral axis should be the largest with the value equal to the maximal shearing stress, which is the reason why FBG $i 1$ and FBG $i 2$ are at the middle height of spoke $i$ with identical angle $+135^{\circ}$ and $+45^{\circ}$ respectively along the direction of the neutral axis of the spoke $i$. 


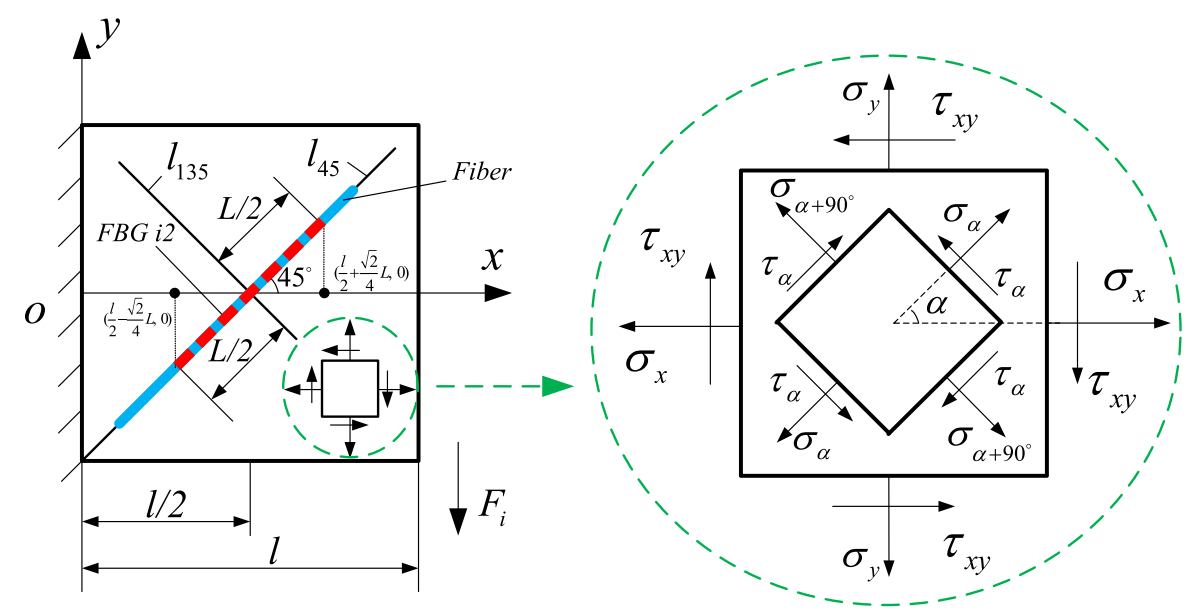

Fig. 2. Stress analysis on the side-surface of the spoke

According to the generalized Hooke's law, strain at the middle point of the FBG sensors in its axial direction can be obtained. While the FBG grating region is $10 \mathrm{~mm}$ long. To obtain accurate strain detected by FBG sensors, strain distribution of the spoke side-surface in the direction of $+135^{\circ}$ and $+45^{\circ}$ along the neutral axis needs to be studied. According to the elastic theory, as shown in Fig. 2, normal stress $\sigma_{\alpha}$ can be expressed as:

$$
\sigma_{\alpha}=\sigma_{x} \cos ^{2} \alpha+\sigma_{y} \sin ^{2} \alpha+2 \tau_{x y} \sin \alpha \cos \alpha
$$

Substituting Eqs. (2), (3) and $\sigma_{y}=0$ into Eq. (4), the normal stress in the direction of $+135^{\circ}$ and $+45^{\circ}$ along the neutral axis can be obtained as follows:

$$
\begin{aligned}
\sigma_{135} & =\frac{F_{i}}{2 I}(l-x) y-\frac{F_{i}}{I}\left(\frac{h^{2}}{8}-\frac{y^{2}}{2}\right) \\
\sigma_{45} & =\frac{F_{i}}{2 I}(l-x) y+\frac{F_{i}}{I}\left(\frac{h^{2}}{8}-\frac{y^{2}}{2}\right)
\end{aligned}
$$

According to the generalized Hooke's law, the normal strain in the direction of $+135^{\circ}$ and $+45^{\circ}$ along the neutral axis can be calculated by the following:

$$
\begin{aligned}
\varepsilon_{135} & =\frac{1+\mu}{E}\left[(1-\mu) \sigma_{135}-\mu \sigma_{45}\right] \\
\varepsilon_{45} & =\frac{1+\mu}{E}\left[(1-\mu) \sigma_{45}-\mu \sigma_{135}\right]
\end{aligned}
$$

Where $E$ is the Young's modulus, $\mu$ is the Poisson's ratio of material. Substituting Eqs. (5) and (6) into Eqs. (7) and (8), yields:

$$
\begin{aligned}
& \varepsilon_{135}(x, y)=\frac{6(1+\mu)}{E b h^{3}}\left[y^{2}+(1-2 \mu)(l-x) y-\frac{h^{2}}{4}\right] F_{i} \\
& \varepsilon_{45}(x, y)=\frac{6(1+\mu)}{E b h^{3}}\left[-y^{2}+(1-2 \mu)(l-x) y+\frac{h^{2}}{4}\right] F_{i}
\end{aligned}
$$

As shown in Fig. 2, the length of the FBG grating region is $L$. FBG $i 2$ is on the straight line $l_{135}: y=x-l / 2$, more specifically is on the curve $\Gamma_{2}: y=x-l / 2$, $(l / 2-\sqrt{2} L / 4 \leq x \leq l / 2+\sqrt{2} L / 4)$. FBG $i 1$ is on the straight line $l_{45}: y=$ $-x+l / 2$, more specifically is on the curve $\Gamma_{1}: y=-x+l / 2,(l / 2-\sqrt{2} L / 4 \leq$ $x \leq l / 2+\sqrt{2} L / 4)$. Average strain detected by FBG $i 1$ and FBG $i 2$ respectively are: 


$$
\begin{aligned}
& \varepsilon_{F B G i 1}=\frac{\int_{\Gamma_{1}} \varepsilon_{135}(x, y) d s}{L} \\
& =\frac{\int_{\frac{l}{2}-\frac{\sqrt{2}}{4} L}^{\frac{l}{2}+\frac{\sqrt{2}}{4} L}\left[(-x+l / 2)^{2}+(1-2 \mu)(l-x)(-x+l / 2)-\frac{h^{2}}{4}\right] \sqrt{2} d x}{L} \frac{6(1+\mu)}{E b h^{3}} F_{i} \\
& =\left(\frac{1-\mu}{2} L^{2}-\frac{3 h^{2}}{2}\right) \frac{(1+\mu)}{E b h^{3}} F_{i} \\
& \varepsilon_{F B G i 2}=\frac{\int_{\Gamma_{2}} \varepsilon_{45}(x, y) d s}{L} \\
& =\frac{\int_{\frac{1}{2}-\frac{\sqrt{2}}{4} L}^{\frac{l}{2}+\frac{\sqrt{2}}{4} L}\left[-(x-l / 2)^{2}+(1-2 \mu)(l-x)(x-l / 2)+\frac{h^{2}}{4}\right] \sqrt{2} d x}{L} \frac{6(1+\mu)}{E b h^{3}} F_{i} \\
& =\left(\frac{\mu-1}{2} L^{2}+\frac{3 h^{2}}{2}\right) \frac{(1+\mu)}{E b h^{3}} F_{i}
\end{aligned}
$$

The Bragg wavelength shift of FBG $\Delta \lambda$ is simultaneously modulated by strain and temperature. The wavelength variation response to axial strain change $\Delta \varepsilon$ and temperature change $\Delta T$ could be given by [13]:

$$
\frac{\Delta \lambda}{\lambda}=\left(1-p_{e}\right) \Delta \varepsilon+\left(\alpha_{f}+\xi\right) \Delta T
$$

where $\lambda$ is the initial wavelength of FBG, $p_{e}, \alpha_{f}$ and $\xi$ is respectively the effective photo-elastic coefficient, the thermal expansion coefficient and the thermal-optic coefficient of fused silica fiber. Therefore, the influence of temperature fluctuation should be eliminated in order to obtain pure strain variation. Assuming the strain transmission ratio is 1, regardless of the effect of adhesive, from Eqs. (11), (12) and (13), the relation of wavelength shifts of FBG $i 1$ and FBG $i 2$ with the force $F_{i}$ $(i=1,2,3)$ can be expressed as follows:

$$
\begin{aligned}
& \frac{\Delta \lambda_{i 1}}{\lambda_{i 1}}=\left(1-p_{e}\right)\left(\frac{1-\mu}{2} L^{2}-\frac{3 h^{2}}{2}\right) \frac{(1+\mu)}{E b h^{3}} \Delta F_{i}+\left(\alpha_{f}+\xi\right) \Delta T \\
& \frac{\Delta \lambda_{i 2}}{\lambda_{i 2}}=-\left(1-p_{e}\right)\left(\frac{1-\mu}{2} L^{2}-\frac{3 h^{2}}{2}\right) \frac{(1+\mu)}{E b h^{3}} \Delta F_{i}+\left(\alpha_{f}+\xi\right) \Delta T
\end{aligned}
$$

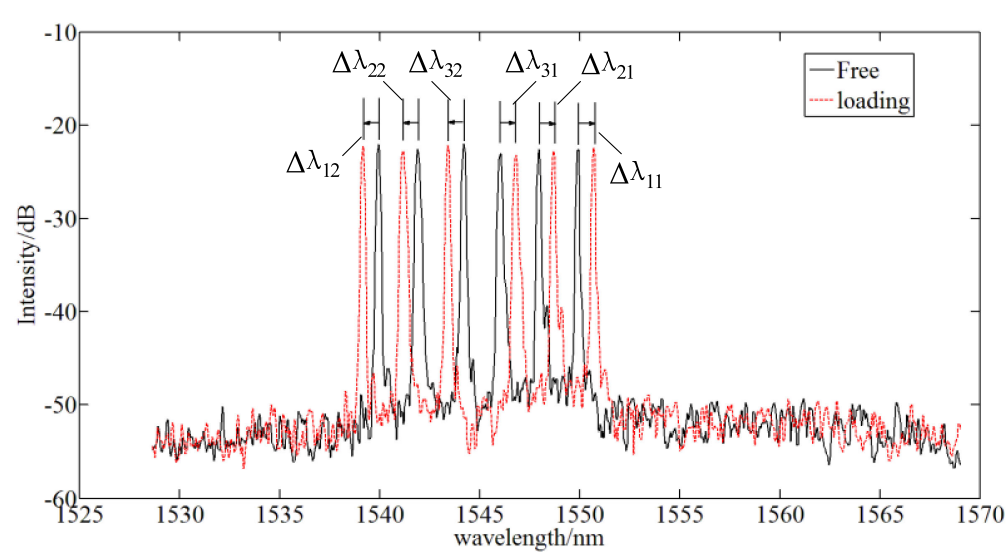

Fig. 3. Reflected signal of the fabricated FBG force transducer 
In Eqs. (14) and (15) $\lambda_{i 1}$ and $\lambda_{i 2}$ is respectively the initial wavelength of FBGi1 and FBG $i 2$. Temperature change $\Delta T$ cause initial wavelengths $\lambda_{i 1}$ and $\lambda_{i 2}$ increase, however, force load $F_{i}$ makes the $\lambda_{i 1}$ increase and $\lambda_{i 2}$ decrease as shown in Fig. 3 . In this force sensor the 6 FBGs are fabricated with approximate initial wavelengths $\left(\lambda_{11}=1540 \mathrm{~nm}, \lambda_{12}=1546 \mathrm{~nm}, \lambda_{21}=1542 \mathrm{~nm}, \lambda_{22}=1544 \mathrm{~nm}, \lambda_{31}=1548 \mathrm{~nm}\right.$, $\left.\lambda_{32}=1550 \mathrm{~nm}\right)$. The initial wavelengths $\left(\lambda_{11}, \lambda_{12}, \lambda_{21}, \lambda_{22}, \lambda_{31}, \lambda_{32}\right)$ are much larger than the wavelength shifts $\left(\Delta \lambda_{11}, \Delta \lambda_{12}, \Delta \lambda_{21}, \Delta \lambda_{22}, \Delta \lambda_{31}, \Delta \lambda_{32}\right)$, so that $\lambda_{11}, \lambda_{12}, \lambda_{21}$, $\lambda_{22}, \lambda_{31}, \lambda_{32}$ could be replaced by an equivalence value $\lambda_{0}$. As the distance between the 6 FBGs is small, they are assumed to undergo the same temperature variation. With these two assumptions the difference between the two wavelength shifts could be adapted by subtracting Eq. (14) from Eq. (15):

$$
\Delta \lambda_{i 1}-\Delta \lambda_{i 2}=\frac{\lambda_{o}(1+\mu)\left(1-p_{e}\right)\left[(1-\mu) L^{2}-3 h^{2}\right]}{E b h^{3}} \Delta F_{i}
$$

Rewriting Eq. (16) as:

$$
\begin{gathered}
\Delta \lambda_{i 1}-\Delta \lambda_{i 2}=k \Delta F_{i} \\
k=\lambda_{o}(1+\mu)\left(1-p_{e}\right)\left[(1-\mu) L^{2}-3 h^{2}\right] / E b h^{3}
\end{gathered}
$$

Where $k$ is the force sensitivity and is a constant for any given $b, h$ and $L$ value. So the total wavelength shift of the 3 pair FBGs is:

$$
\Delta \lambda=\sum_{i=1}^{3}\left(\Delta \lambda_{i 1}-\Delta \lambda_{i 2}\right)=\sum_{i=1}^{3} k \Delta F_{i}=k \Delta F
$$

Eq. (19) shows that total wavelength shift difference $\Delta \lambda$ of the 6 FBGs is a linear function of force $F$ and independent of temperature variation. In addition, when the wavelength shift difference of the 6 FBGs is used as sensing signal, the force sensitivity is increased compared to using the wavelength shift of one single FBG as sensing signal from Eqs. (14), (15) and (16).

To verify the theoretical model, a 3 -spoke-type spring element specimen is manufactured by using 304 stainless steel. The dimensions and mechanical properties of theoretical model are given in Table I. Summiting these values to Eq. (18), the theoretical force sensitivity of the force transducer can be obtained: $k=$ $87.77 \mathrm{pm} / \mathrm{kN}$.

Table I. Dimensions and mechanical properties of theoretical model

\begin{tabular}{c|c|c|c}
\hline Dimensions properties & Values & Mechanical properties & Values \\
\hline Length of the spoke $l$ & $25 \mathrm{~mm}$ & Young's modulus of spoke $E$ & $1.93 \mathrm{e} 5 \mathrm{MPa}$ \\
\hline Width of the spoke $b$ & $10 \mathrm{~mm}$ & Poisson's ratio of spoke $\mu$ & 0.3 \\
\hline Height of the spoke $h$ & $25 \mathrm{~mm}$ & $\begin{array}{c}\text { effective photo-elastic } \\
\text { coefficient of the FBGs } P_{e}\end{array}$ & 0.22 \\
\hline $\begin{array}{c}\text { Length of the FBG } \\
\text { grating region } L\end{array}$ & $10 \mathrm{~mm}$ & $\begin{array}{c}\text { equivalence initial wavelength } \\
\text { value of the 6 FBGs } \lambda_{o}\end{array}$ & $1550 \mathrm{~nm}$ \\
\hline
\end{tabular}

\section{Simulation analysis}

\subsection{Strain analysis}

To verify the feasibility of the proposed method and structure, theoretical calculation by Matlab software and finite element method (FEM) with ANSYS of spoke- 
type spring element has been carried out. Fig. 4 shows the theoretical calculation results based on Eqs. (10) and (11) using Matlab software and the simulated equivalent strain distribution along FBG i1 and FBG i2 grating region of the spoke by FEM. The structure parameters of spoke used in the calculation and simulation are set as section 2 and the force is $F=-50 \mathrm{kN}$ in the simulation, $F_{1}=F_{2}=$ $F_{3}=-50 / 3 \mathrm{kN}$ in the theoretical calculation. It could be found from Fig. 4 that the theoretical calculation and FEM analysis results are similar, both indicating that there exits strain gradients on the FBG grating region of the spokes. And the FEM analysis results is more smooth than the theoretical calculation results, for the small deformation of the inner and outer shell reduces the strain gradients of the spoke. While to obtain an accurate sensing model of the force transducer, the influence of strain gradients should be considered.

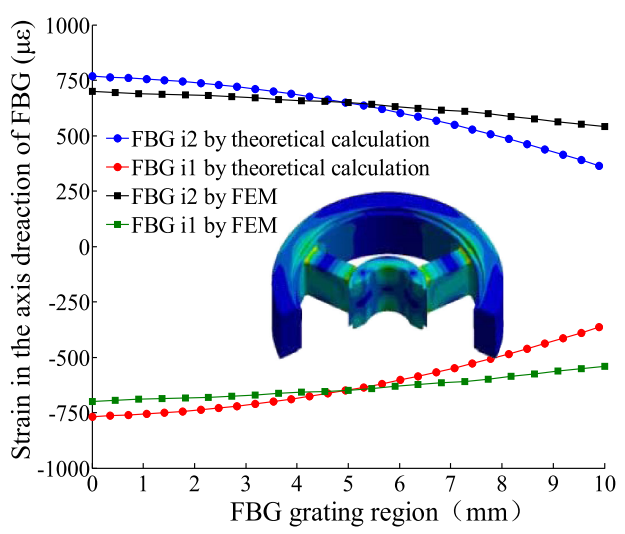

Fig. 4. Strain distribution along FBG i1 and FBG i2 grating region of the spoke

\subsection{Dynamic characteristics simulation analysis}

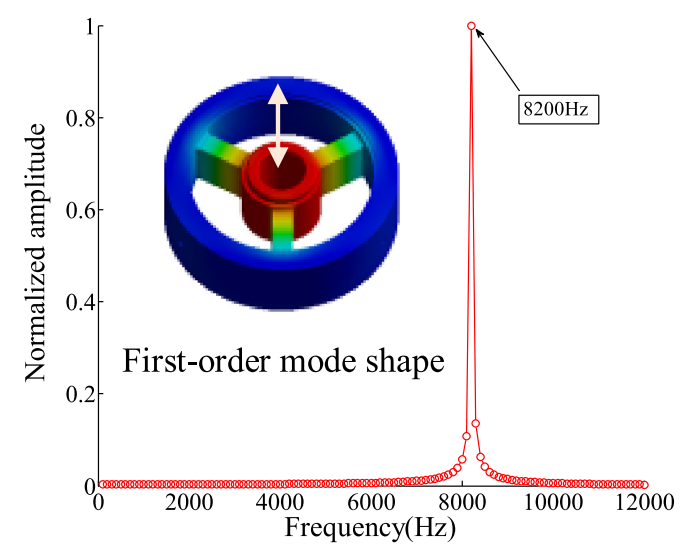

Fig. 5. Harmonic response of the ANSYS simulation

Force transducers are generally required to possess good dynamic performance to accurately obtain the characteristic of the force to be measured. The dynamical characteristic of the spoke-type spring element is analyzed by modal analysis and harmonic response analysis with ANSYS. The modal analysis results show that the inherent frequency of the spoke-type spring element is $8212 \mathrm{~Hz}$. Fig. 5 shows the 
maximum deformation of the spoke-type spring element under harmonic force with $-50 \mathrm{kN}$ amplitude and $0 \sim 12000 \mathrm{~Hz}$ frequency range (the interval is $50 \mathrm{~Hz}$ ). The maximum deformation occurs when the frequency is near the inherent frequency and the first-mode shape is vertical vibration.

\section{Experimental analysis of sensing properties}

\subsection{Static properties}

The schematic diagram of the experimental setup and instruments are shown in Fig. 6. The designed FBG force transducer is placed on press machine $(100 \mathrm{kN} /$ MTS810) which is used to control the force applied on the sensor accurately. An FBG interrogator with minimum resolution of $1 \mathrm{pm}$ is used to monitor the Bragg wavelength shift of the 6 FBGs under different force. The FBG wavelength interrogation system is mainly composed of a FBG interrogator and related ancillary components such as multiplexers, switches and software. The FBG interrogator shown in Fig. 6 is based on the tunable Fabry-Perot filter technology with sampling rate of $100 \mathrm{~Hz}$. During the experiment, the force applied on the designed force transducer by the press machine is changed from 0 to $50 \mathrm{kN}$ with a step of $5 \mathrm{kN}$ and the temperature is fixed at the room temperature.

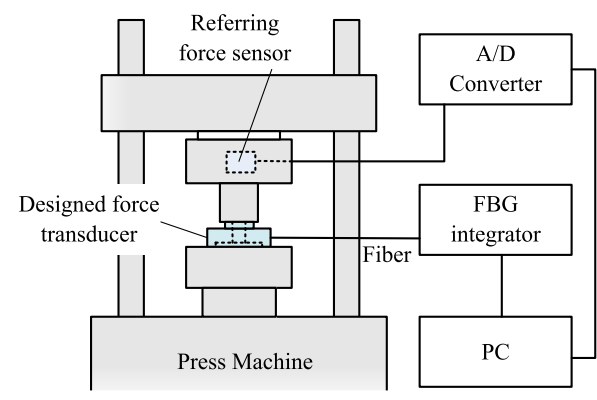

(a) Schematic diagram

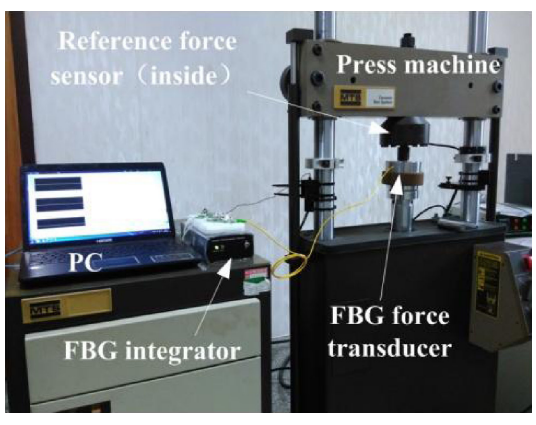

(b) Experiment instruments images

Fig. 6. Experimental setup

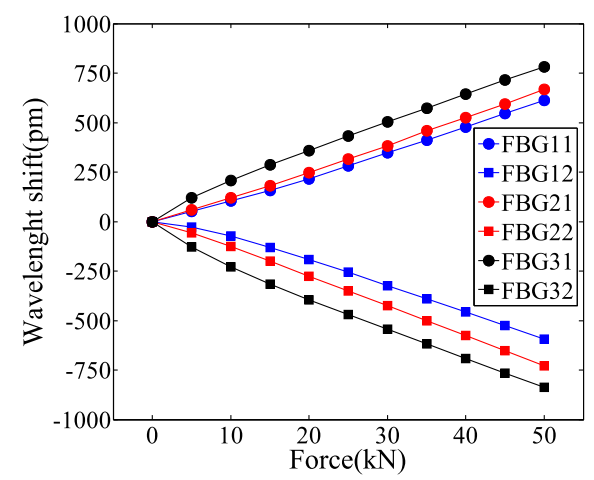

Fig. 7. Wavelength shifts of the 6 FBGs versus force variation

Fig. 7 shows the wavelength shifts versus force variation for the 6 FBGs. FBG $i 1(\mathrm{i}=1,2,3)$ are stretched and senses the positive strain. While FBG $i 2$ $(i=1,2,3)$ are compressed and senses the negative strain. All the variation patterns are linear. Fig. 8(a) lists the total wavelength shift difference values $\Delta \lambda$ of the 6 FBGs at each force value. The experiment has been repeated for 6 times with the 


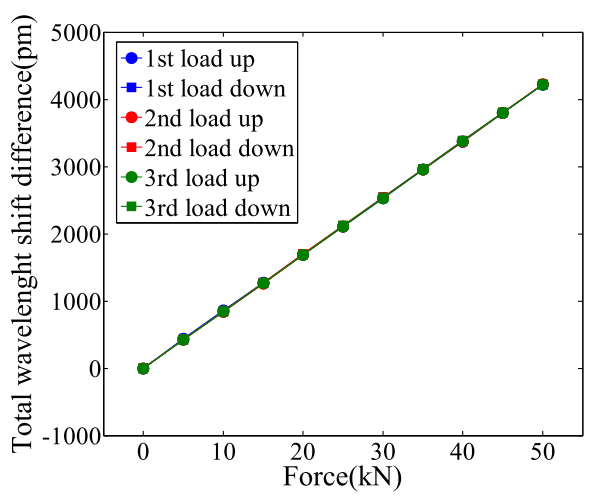

(a) Repeatability experiment

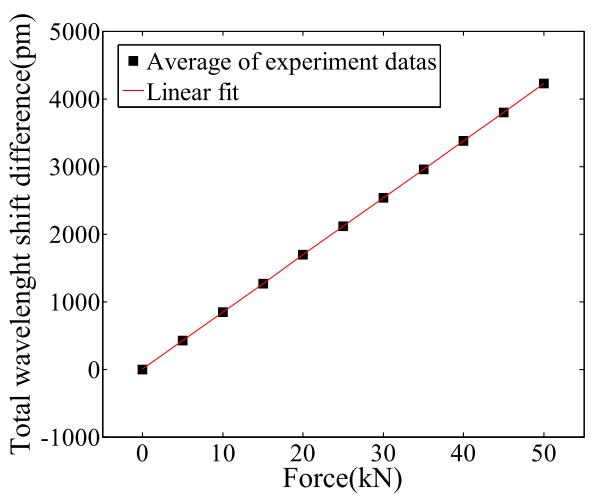

(b) Linear fitting

Fig. 8. Variation of the total wavelength shift difference of the 6 FBGs versus force

force load being firstly increased and then decreased to demonstrate the repeatability of the designed force transducer. The repeatability error is $5 \mathrm{pm}$ which is less than $0.12 \%$. Fig. $8(\mathrm{~b})$ is the variation of average wavelength shift differences obtained in the repeated experiments. The fitting results are also presented and the force sensitivity is $84.43 \mathrm{pm} / \mathrm{kN}$ and the fitting linear correlation coefficient reaches $99.99992 \%$. This force sensitivity shows strong consistency with the theoretical sensitivity $87.77 \mathrm{pm} / \mathrm{kN}$. The actual test values is a bit smaller than theoretical values, one of the most important factors is that the strain transfer efficiency from the spoke surface to the fiber core through the adhesives and fiber cladding is less than $100 \%$. A little part of the strain energy is stored in the adhesive and fiber cladding. As the resolution and precision of the FBG integrator is $1 \mathrm{pm}$ and $\pm 5 \mathrm{pm}$ respectively, the resolution and precision of the developed FBG transducer is $11.8 \mathrm{~N}$ and $\pm 59 \mathrm{~N}$, thus $0.24 \% \mathrm{FS}$ and $0.12 \% \mathrm{FS}$.

\subsection{Dynamic properties}

Hammering method is utilized to test the dynamic measurement frequency range of the force sensor. The testing system is shown in Fig. 9, including a hammer (B\&K

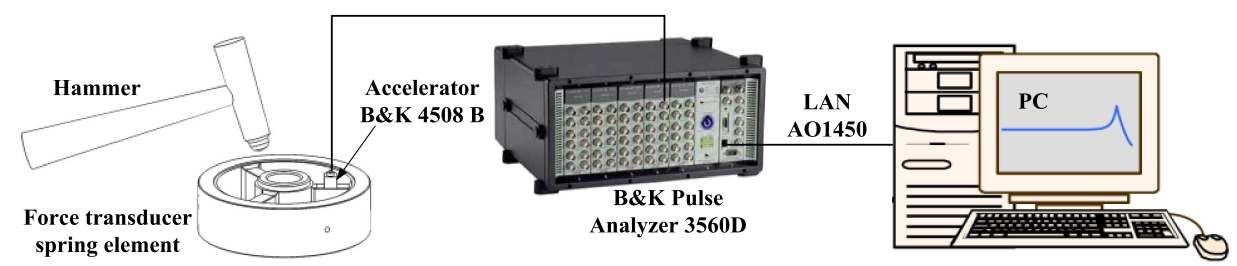

Fig. 9. Natural frequency test setup

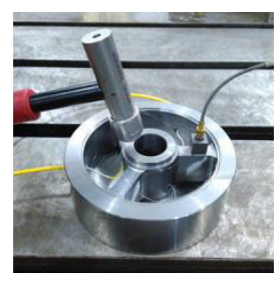

(a) Freely on the foundation

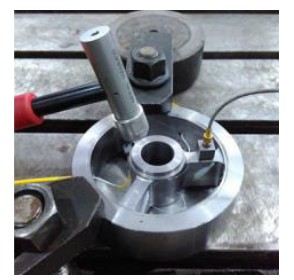

(b) Fixed on the foundation

Fig. 10. Boundary constraints 
EE-0359), an acceleration sensor (B\&K 4508B), multi-analyzer system (B\&K pulse analyzer $3560 \mathrm{D}$ ), and computer with vibration analysis software (B\&K pulse software 7700). Boundary conditions of the force sensor directly influence its modal test results. As shown in Fig. 10, under two kinds of installation the dynamic performance of the sensor is measured. In Fig. 10(a) the force sensor is placed on the solid foundation freely, while in Fig. 10(b) the outer shell of the force sensor is fixed on the ground. The acceleration sensor (B\&K 4508 B) is installed on the up surface of a spoke. With a hammer knocking the inner shell of the force sensor vertically, the acceleration amplitude of the spoke in the vertical direction is measured. The frequency spectrum is obtained by Fourier transform, as shown in Fig. 11(a). When the outer shell is free, the first order natural frequency is $8802 \mathrm{~Hz}$, when the outer shell is fixed, the first order natural frequency increases slightly, to $8816 \mathrm{~Hz}$. The test result is in conformity with the theoretical calculation value of $8212 \mathrm{~Hz}$, namely the dynamic measurement range of the sensor is wide. Within the range of $0 \sim 100 \mathrm{~Hz}$, its performance is better.

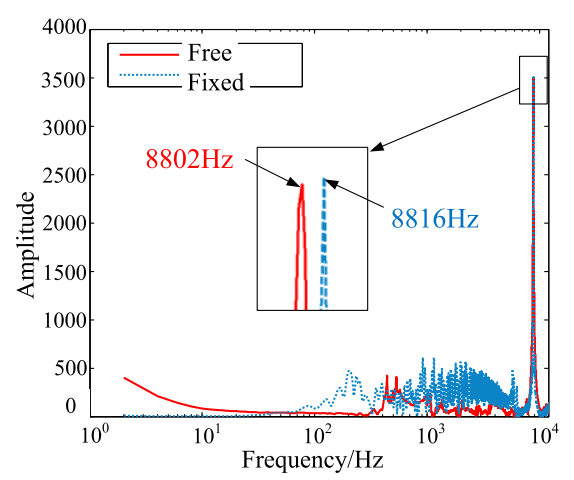

(a) Natural frequency test

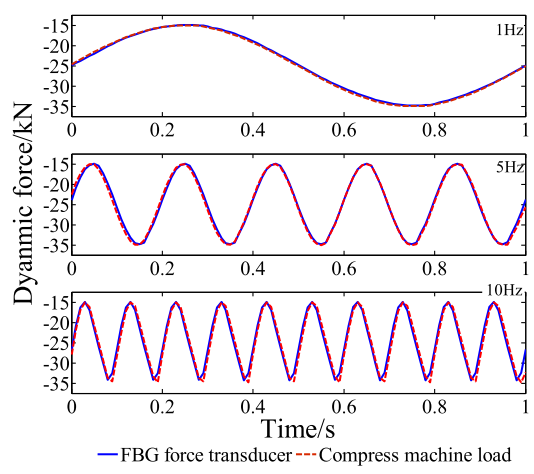

(b) Dynamic force measurement

Fig. 11. Dynamic properties measurement

To further verify the dynamic performance of the force sensor, the load of the compress machine is set as $F=-25+10 \sin 2 \pi f \mathrm{t}(\mathrm{kN})$. Adjusting the load frequency $f$ as $1 \mathrm{~Hz}, 5 \mathrm{~Hz}$ and $10 \mathrm{~Hz}$ respectively, Fig. 11(b) shows the testing force by designed force transducer and it can accurately measure the dynamic load on the inner shell. For the practical engineering, the dynamic frequency of heavy load is generally not high, the force sensor can satisfy most dynamic load test.

\subsection{Temperature-independent properties}

In order to evaluate the sensor's temperature compensation performance, the variation of wavelength shift difference versus temperature increase has been tested. During the experiment a thermostat is used to change the surrounding temperature of the sensor. The temperature is changed between $30^{\circ} \mathrm{C}$ to $120^{\circ} \mathrm{C}$ and the sampling interval is $10^{\circ} \mathrm{C}$. The wavelength shift difference is listed in Fig. 12 . The fluctuation of wavelength shift is mainly caused by the difference of the thermal stress distributed on the spoke's surface where the FBGs are bonded. The maximum average difference value is only $6.57 \mathrm{pm}$ and the sensor's temperature compensation effect is conspicuous. 


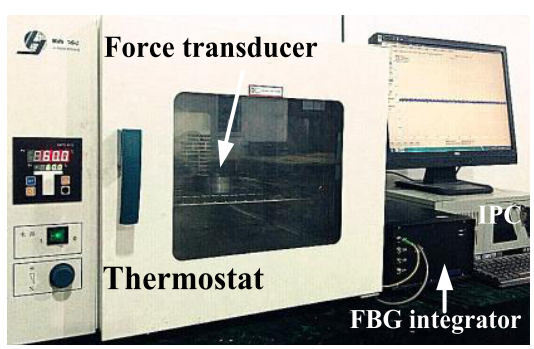

(a) Temperature influence testing field

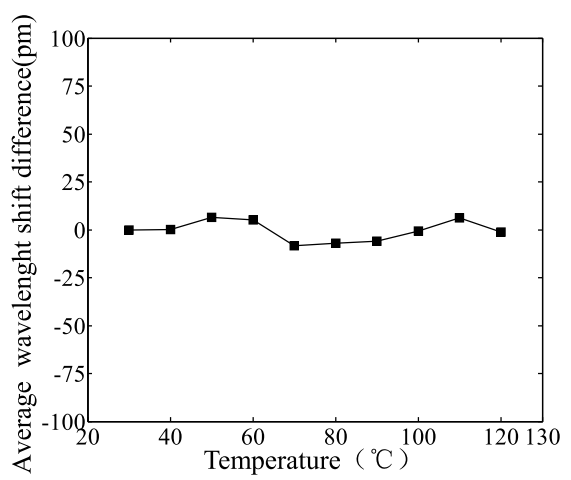

(b) Testing result

Fig. 12. The average wavelength shift difference of the 6 FBGs versus the surrounding temperature

\section{Conclusion}

A FBG force transducer with temperature compensation has been presented and realized. The sensor adopts 3 spokes each with 2 FBGs adhered on the two sidesurfaces of the spoke as the force sensitive component.

1) Through calculating strain distribution in the FBG grating region accurately and then using curvilinear integral algorithm to calculate the average strain FBG measured, the established mathematical sensitivity $87.77 \mathrm{pm} / \mathrm{kN}$ of this novel sensor is consistency with the experimental result $84.43 \mathrm{pm} / \mathrm{kN}$;

2) The sensor shows good static and dynamic performance. Using the FBGs' wavelength shift difference as sensing signal, the measurement sensitivity is enhanced to twice higher than using single FBG. The linearity of the sensor is good with a fitting linear correlation coefficient of $99.99992 \%$ and the repeatability error is less than $0.12 \%$. The resolution and precision of the developed FBG transducer is $0.24 \% \mathrm{FS}$ and $0.12 \% \mathrm{FS}$. In addition, it is insensitive to partial load, and its dynamic test range is wide;

3) More important, the temperature cross-sensitivity is effectively avoided by using the wavelength shift difference between the 6 FBGs as the force sensitive parameter.

The measure range and sensitivity can be easily adjusted by simply optimizing the size and material of the spring element to adapt different measure demands, and multiplexing could be realized by adding the FBGs on a single fiber. With these merits this kind of force sensor is supposed to have many potential applications for quasi-distributed force measurement in advanced electromechanical devices or heavy-duty engineering structure.

\section{Acknowledgments}

This work was supported by the Key Project of National Nature Science Foundation of China under Grant no. 51475343 and the International Science \& Technology Cooperation Program of China under Grant no. 2015DFA70340. 